\title{
BMI and healthy life expectancy in old and very old women
}

\author{
L. Leigh $^{1 *}$, J. E. Byles ${ }^{1}$ and C. Jagger ${ }^{2}$ \\ ${ }^{1}$ Research Centre for Generational Health and Ageing, University of Newcastle, University Drive, Callaghan, NSW 2308, Australia \\ ${ }^{2}$ Institute of Health \& Society, Newcastle University Institute for Ageing, Newcastle University, Newcastle upon Tyne NE4 5PL, UK \\ (Submitted 22 January 2016 - Final revision received 11 May 2016 - Accepted 20 May 2016 - First published online 16 June 2016)
}

\section{Abstract}

There is conflicting evidence for the effect of BMI on mortality at older ages, and little information on its effect on healthy life expectancy (HLE). Longitudinal data were from the 1921-1926 cohort of the Australian Longitudinal Study on Women's Health ( $n$ 11 119), over 18 years of follow-up. Self-rated health status was measured at each survey, and BMI was measured at baseline. Multi-state models were fitted to estimate the effect of BMI on total life expectancy (TLE) and HLE. Compared with women of normal weight, overweight women at the age of 75 years had similar TLE but fewer years healthy $(-0.79 ; 95 \% \mathrm{CI}-1 \cdot 21,-0 \cdot 37)$ and more years unhealthy $(0 \cdot 99 ; 95 \%$ CI $0.56,1.42)$. Obese women at the age of 75 years lived fewer years in total than normal-weight women $(-1 \cdot 09 ; 95 \%$ CI $-1 \cdot 77,-0 \cdot 41)$, and had more unhealthy years (1.46; $95 \%$ CI 0.97, 1.95 years). Underweight women had the lowest TLE and the fewest years of healthy life. Women should aim to enter old age at a normal weight and in good health, as the slight benefit on mortality of being overweight is offset by spending fewer years healthy. All outcomes were better for those who began in good health. The relationship between weight and HLE has important implications for nutrition for older people, particularly maintenance of lean body mass and prevention of obesity. The benefit of weight loss in obese older women remains unclear, but we support the recommendation that weight-loss advice be individualised, as any benefits may not outweigh the risks in healthy obese older adults.

\section{Key words: BMI: Healthy ageing: Older persons: Mortality}

Obesity is a global epidemic, and the World Health Organization recommends that all persons maintain a normal $\mathrm{BMI}^{(1)}$. However, there is ongoing discussion as to whether the WHO recommendations should apply to old (70-79 years) and very old ( $\geq 80$ years) persons ${ }^{(2-4)}$. Among the older population, those who are underweight generally have a higher risk of death than those of normal weight ${ }^{(5,6)}$, and some studies report a survival advantage for people in the overweight range ${ }^{(7-9)}$.

These studies of the effect of BMI at older ages emphasise mortality, rather than quality of life or disability. However, obesity is also associated with an increased risk of CVD, cancer, hypertension, stroke, diabetes and other medical problems $^{(5,10,11)}$, and weight loss in older persons may reduce morbidity from these diseases ${ }^{(12-15)}$. An alternative measure that incorporates both quality and quantity of remaining life is healthy life expectancy (HLE), which estimates the expected remaining number of years of life spent in a state of good health. Life expectancies continue to increase ${ }^{(16)}$, and research into HLE, as opposed to total life expectancy (TLE), helps to answer the crucial question of "whether the extra years gained year on year in life expectancy are healthy years ${ }^{(17)}$. Given the rapidly expanding ageing population ${ }^{(18)}$, there is growing interest in investigating the predictors of HLE in ageing populations, as well as $\operatorname{TLE}^{(17,19-22)}$. Steensma et al. ${ }^{(23)}$ estimated TLE and HLE in adults aged 20-100 years. Although their results were in agreement with many studies in that overweight adults had a slightly longer TLE compared with normal-weight adults, they also found that these adults spent a greater proportion of these extra years in poorer health. Reynolds et al. ${ }^{(24)}$ found that obesity had little effect on TLE in adults aged over 70 years, but that the obese were more likely to become disabled, living a greater proportion of their remaining years with disability, and concluded that obesity-related death is therefore less of a concern than obesity-related disability in this age group. However, another study ${ }^{(3)}$ that looked at adults aged 65 years and over found that although underweight adults had worse outcomes than normal-weight adults, those who were overweight or obese rarely had worse, and sometimes had better, outcomes than normal-weight adults.

One possible explanation for the differing effects found in these studies may be the wide range of ages investigated. The effect of BMI may differ at extreme old age because of the changing relationship between BMI, disease processes and treatments ${ }^{(3)}$.

Effects may also differ by sex $^{(9)}$, and thus sex-specific estimates are also needed. Women live longer than men, and

Abbreviations: HLE, healthy life expectancy; TLE, total life expectancy; UHLE, unhealthy life expectancy.

* Corresponding author: L. Leigh, fax +61 24042 0044, email Lucy.Leigh@newcastle.edu.au 
as the population ages older women will comprise an increasingly larger proportion of the Australian population ${ }^{(25)}$. Older women also make up a majority of the people with conditions such as dementia, sensory impairment and falls, and they are more likely to need aged care services ${ }^{(25)}$. Flicker et al. ${ }^{(9)}$ found that women experienced lower mortality for all categories of BMI than men, and Nagai et al. ${ }^{(5)}$ found that obese women had a higher risk of death than normal-weight women, but the effect was non-significant for men. Therefore, it is important to explore the effect of BMI on TLE and HLE exclusively in a cohort of very old women. The primary aim of this research is to investigate the relationship between BMI, TLE and HLE (as measured by subjective self-rated health), in a longitudinal study of women born in 1921-1926 with 18 years of follow-up. A secondary goal is to investigate the differences in TLE and HLE for obese women who lose weight.

\section{Methods}

Data

Data were from the Australian Longitudinal Study on Women's Health - a nationally representative, prospective study of over 40000 participants that commenced in 1996. Cohorts of women, born in 1973-1978, 1946-1951 and 1921-1926, were sampled from the Medicare Australia database and invited to complete the baseline postal survey. Further details on the establishment of the cohorts, and follow-up, have been published elsewhere ${ }^{(26-28)}$. Following the baseline survey, women were resurveyed on a three-yearly basis, and after 2011 the 1921-1926 cohort was surveyed on a 6-monthly basis. This study presents data collected on the 1921-1926 cohort, who completed the baseline survey in 1996 (survey 1) when they were aged 70-75 years and subsequently followed up over 18 years.

At each of surveys 1 through 6, and the first four 6-monthly surveys (surveys returned before 8 November 2013), women were asked to rate their own health as excellent, very good, good, fair or poor. This rating was used to classify participants' health at each time point as either 'good' (a response of excellent, very good, or good) or 'poor' (a response of fair or poor). BMI was calculated from self-reported weight and height, and classified according to the World Health Organization categories ${ }^{(29)}$ as underweight (BMI $<18.5 \mathrm{~kg} / \mathrm{m}^{2}$ ), normal weight (BMI $\geq 18.5$ and $<25 \mathrm{~kg} / \mathrm{m}^{2}$ ), overweight (BMI $\geq 25$ and $\left.<30 \mathrm{~kg} / \mathrm{m}^{2}\right)$ or obese $\left(\mathrm{BMI} \geq 30 \mathrm{~kg} / \mathrm{m}^{2}\right)$. Death dates were ascertained from the National Death Index (NDI) ${ }^{(30)}$ and censored at the study cut-off date (31 March 2014), this being the last date of reliable death information.

\section{Statistical analysis}

TLE, HLE (time spent in a state of 'good' self-rated health) and unhealthy life expectancies (UHLE; time spent in a state of 'poor' self-rated health) were calculated from modelling the transitions between the three states (good health, poor health and death), over ten surveys (18 years follow-up), using discrete multi-state models implemented in the interpolated Markov chain (IMaCh) software, version $0.98 q^{4}{ }^{(31)}$. IMaCh was designed to estimate health expectancies ${ }^{(32)}$ by estimating an illness-death model, with good health and poor health being 'transient' states (participants can move between these states between surveys) and death an 'absorbing' state ${ }^{(33)}$. IMaCh partitions the time intervals between surveys into shorter steps ${ }^{(21)}$ and then models the transition probabilities using multinomial logistic regression on age (and other covariates) ${ }^{(21)}$. A step-length of 1 month was used to approximate the underlying continuous time process. Missing surveys are accommodated, as the intervals between each survey do not need to be equal ${ }^{(21,22)}$. Baseline BMI was included in the models as a covariate; participants with missing baseline BMI were excluded from the analysis, but a sensitivity analysis was undertaken by backfilling missing baseline BMI with values from later surveys.

Given that weight loss in obese older persons may result in reduced morbidity, a secondary analysis was performed on the subset of obese women, in order to investigate whether weight loss in this group is associated with improved HLE. These women were classified into two groups - those whose BMI category did not change between surveys 1 and 2 and those whose BMI category decreased. TLE, HLE and UHLE were compared for the two groups.

\section{Results}

A total of 12432 women completed the baseline survey. Participants with missing BMI ( $n$ 1313) values were excluded, leaving 11119 women available for analysis, of whom $42 \%$ were still alive at the study cut-off date (31 March 2014) and were censored. A sensitivity analysis was performed to determine the effect of missing BMI by backfilling missing baseline BMI with the nearest reported BMI value (up to survey 4), which recovered 467 women. The results of the sensitivity analysis ( $n$ 11586, not reported) were not substantially different from the original analysis.

Lasagna plots ${ }^{(34)}$ were created (Fig. 1-4) to show the overall proportion of women in each state, and the changes between states, across BMI categories and across the first six surveys. These plots demonstrate that the normal-weight group has the highest proportion of women starting in good health $(74.8 \%)$, whereas the obese group has the smallest proportion (58.1\%). There are few backward transitions from poor to good health (recovery) across any BMI category. The underweight group appears to have the smallest number of transitions from good to poor health, with women being more likely to die than to transition to poor health. In contrast, the obese group appears to have the most transitions from good to poor health over time.

Fig. 5 displays the TLE, HLE and UHLE over time for each BMI category. Expected years of unhealthy life outnumber those of healthy life earliest for obese women (about the age of 82 years), and then about the age of 91 years for overweight women. In contrast, for normal-weight and underweight women, years of HLE exceed years of UHLE at all ages. The data for Fig. 5 are provided in the online Supplementary material.

Overall (regardless of the initial state) TLE at the age of 75 years was not significantly different for normal-weight and 


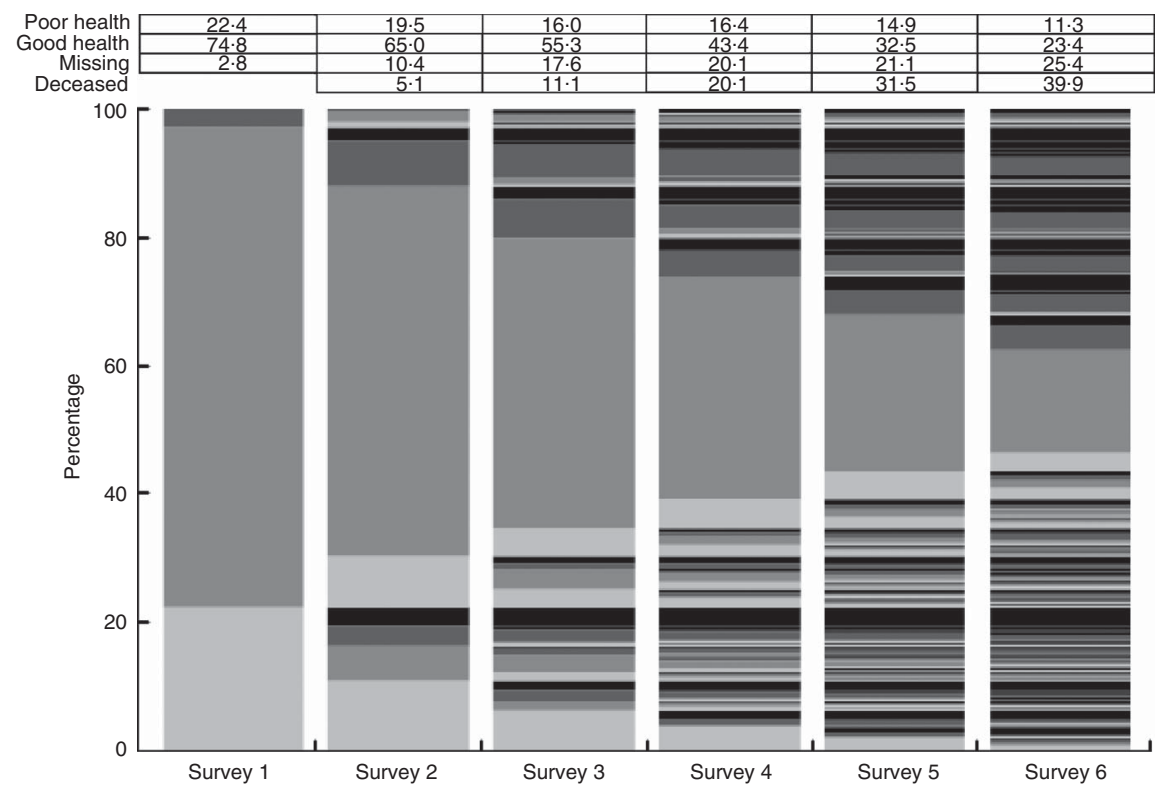

Fig. 1. Lasagna plot for women with normal BMI showing pattern of self-rated health status over surveys 1-6. $\square$, Poor health; $\square$, good health; $\square$, missing; , deceased.

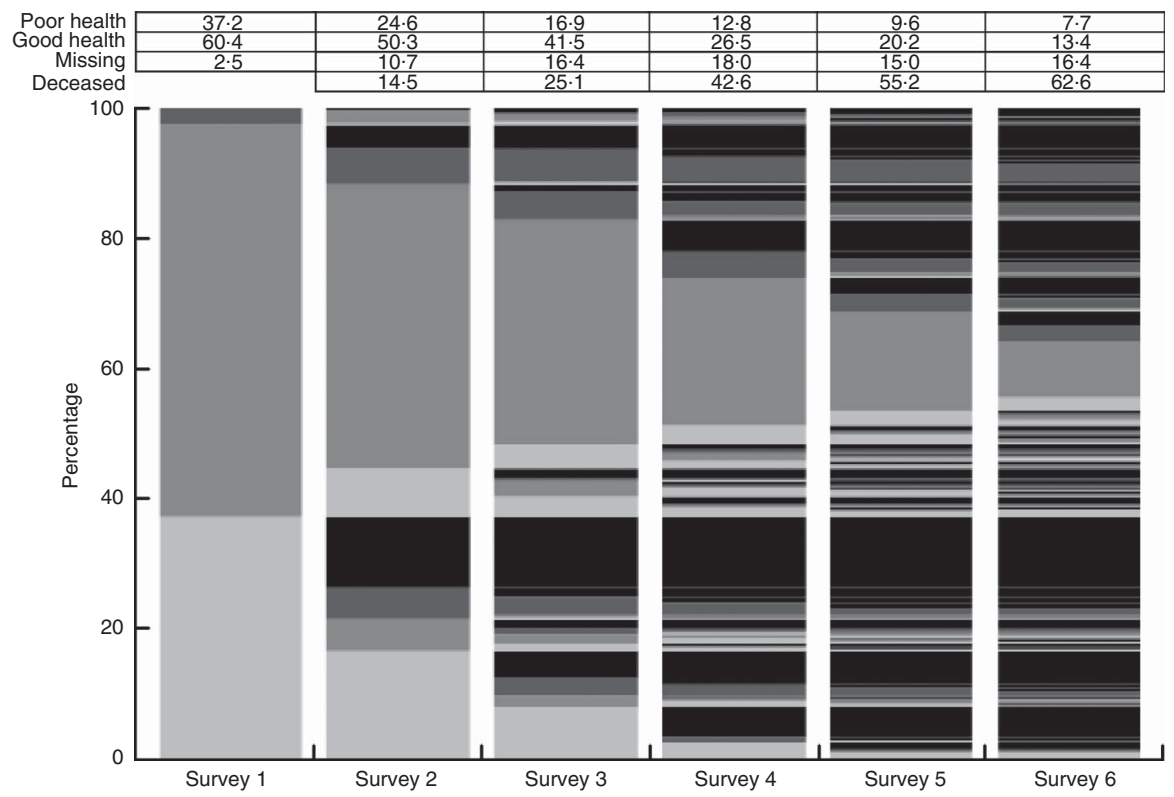

Fig. 2. Lasagna plot for women with underweight BMI showing pattern of self-rated health status over surveys $1-6$. $\square$, Poor health; $\square$, good health; $\square$, missing; 口, deceased.

overweight women (Table 1), although overweight women, compared with those of normal weight, did have significantly more unhealthy years (0.99; $95 \%$ CI $0 \cdot 56,1.42$ years) and fewer healthy ones $(0.79 ; 95 \% \mathrm{CI} 0.37,1.21$ years $)$. Obese women at the age of 75 years also experienced fewer healthy years $(2 \cdot 55$; $95 \%$ CI $2.07,3.03$ years) and more unhealthy years (1.46; $95 \%$ CI $0.97,1.95$ years) than normal-weight women, but in addition they also lived fewer years in total (1.09; 95\% CI 0.41, 1.77 years). Compared with normal-weight women, underweight women had 3.38 (95\% CI 2.43, 4.33) fewer years in total, with 2.46 (95\% CI $1.59,3.33)$ fewer years healthy, although UNHLE at the age of 75 years was not significantly greater.
Table 1 also displays the TLE, HLE and UHLE at the age of 75 years stratified by initial health state (good or poor health). TLE across all BMI categories was greater for women who began in good health than those who began in poor health, with roughly 2 years difference for normal weight, overweight and obese women, and 3 years difference for underweight women. HLE was much greater for those who began in good health compared with those who began in poor health across all BMI categories.

Table 2 displays the effect of BMI on transitioning between good health, poor health and death. Overweight and obese women were more likely to transition from good to poor health 


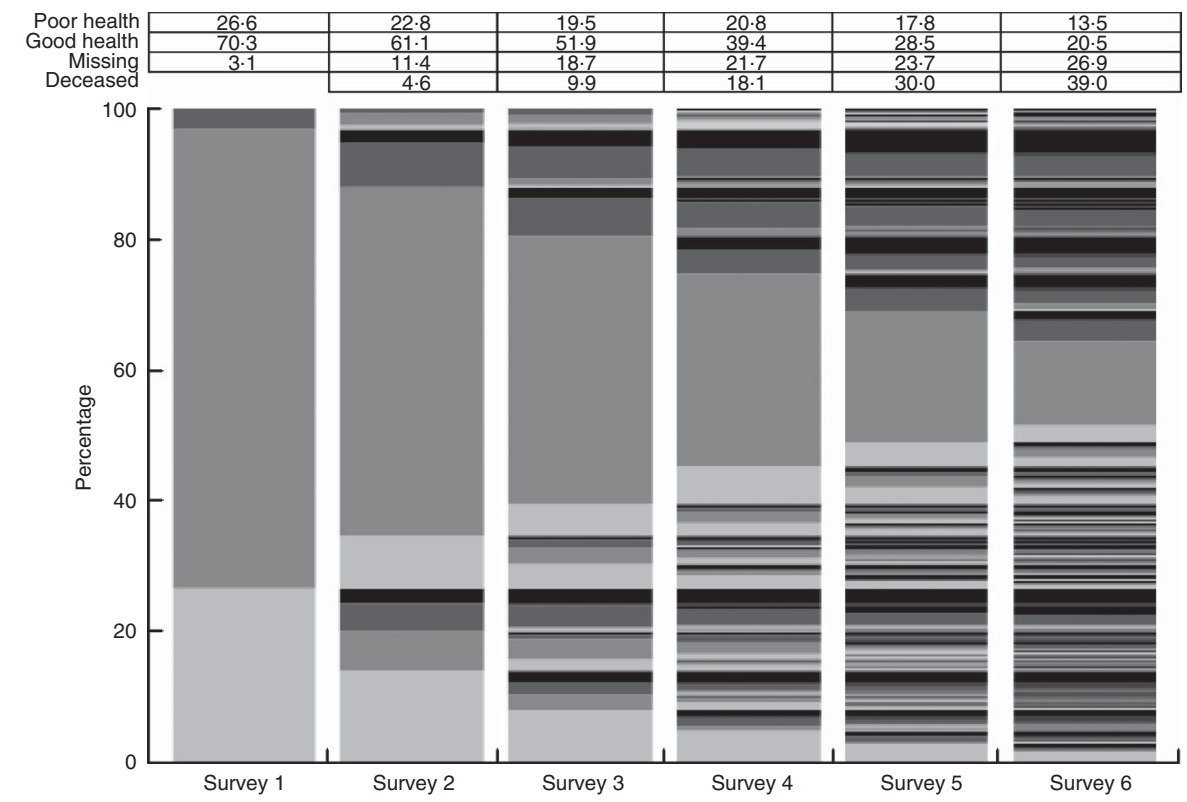

Fig. 3. Lasagna plot for women with overweight BMI showing pattern of self-rated health status over surveys 1-6. $\square$, Poor health; $\square$, good health; $\square$, missing; $\square$, deceased.

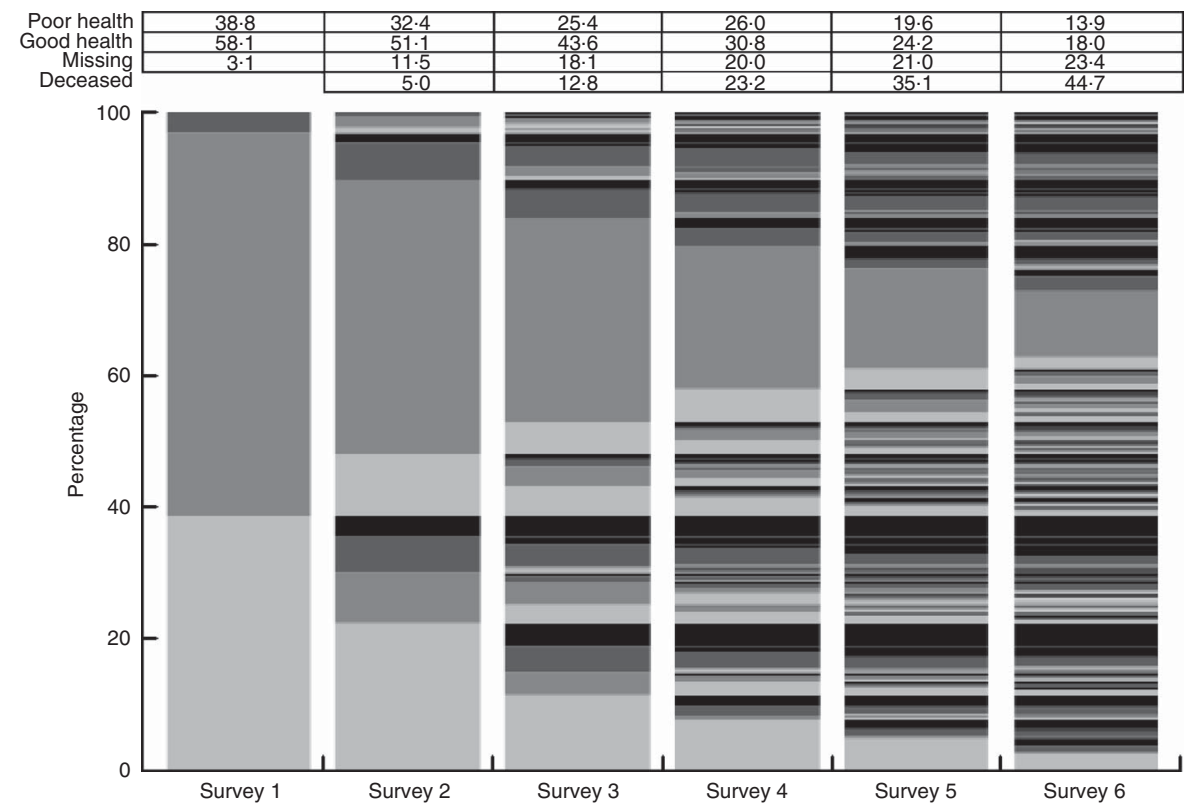

Fig. 4. Lasagna plot for women with obese BMI showing pattern of self-rated health status over surveys $1-6$. $\square$, Poor health; $\square$, good health; $\square$, missing; , deceased.

than normal-weight women (risk ratio (RR) $1 \cdot 15$; $95 \%$ CI 1.08 , 1.23 and RR $1.60 ; 95 \%$ CI $1.46,1 \cdot 75$, respectively), whereas the risk for underweight women was not significantly different. There was no difference in the risk of transitioning from good health to death for any BMI category, nor was there any difference in the risk of recovery. However, underweight women were almost twice as likely to die (from poor health) than normalweight women (RR 1.90; 95\% CI 1.58, 2.28), whereas overweight women were slightly less likely to die (RR 0.84; $95 \%$ CI 0.76, 0.91). There was no difference between normal-weight and obese women in the risk of transitioning from poor health to death.
In order to exclude the possibility that the increased risk of mortality for underweight women starting in poor health was because of pre-existing terminal disease, a second sensitivity analysis was performed, based on the sub-group of women who had survived to at least survey 2 ( $n$ 10660). Any underweight women in this sub-sample had therefore been underweight for at least 3 years, which removes the possibility that their underweight status is a recent change resulting from terminal disease. The results of the sensitivity analysis (not reported) showed no substantive difference from the original analysis. 

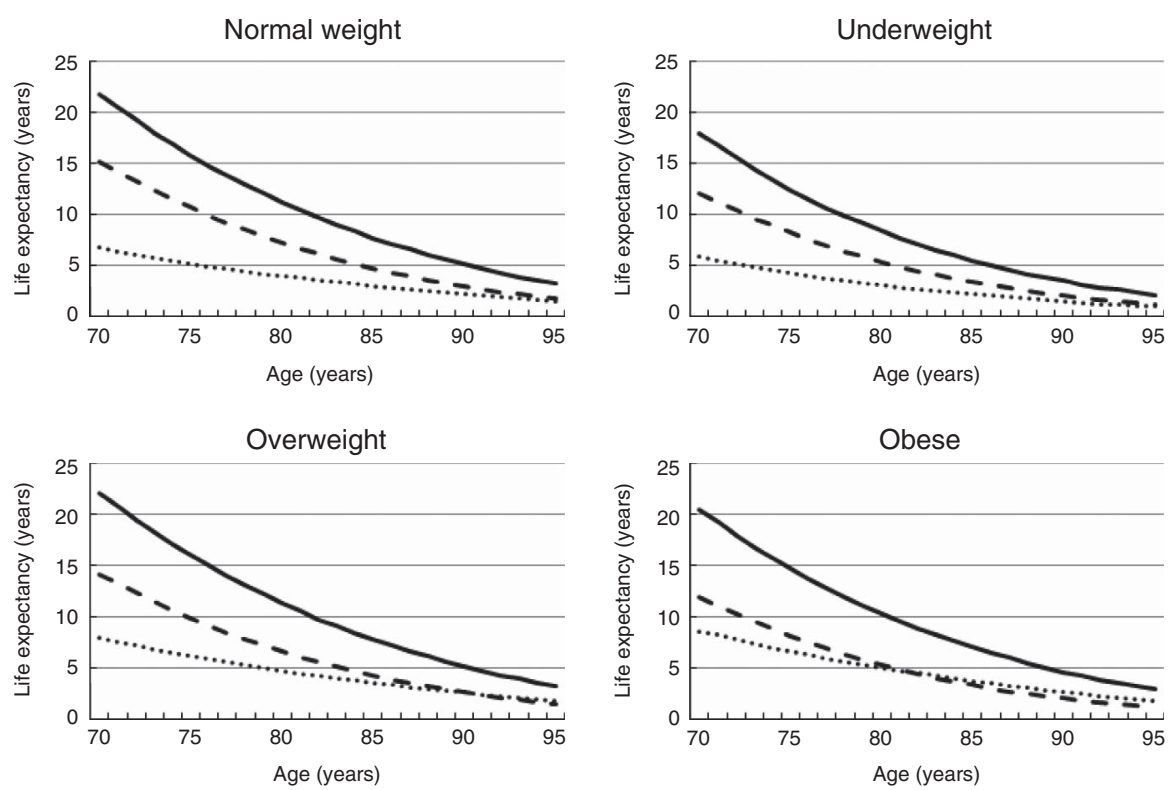

Fig. 5. Total life expectancy (TLE), healthy life expectancy (HLE) and unhealthy life expectancy (UHLE) by BMI category. ——, TLE; — —, HLE; ........., UHLE.

Table 1. Total life expectancy (TLE), healthy life expectancy (HLE) and unhealthy life expectancy (UHLE) (years) at the age of 75 years by BMI, overall and by initial state

(Years and $95 \%$ confidence intervals)

\begin{tabular}{|c|c|c|c|c|c|c|c|c|}
\hline & \multicolumn{2}{|c|}{ Normal weight $(50.1 \%)$} & \multicolumn{2}{|c|}{ Underweight (3.2\%) } & \multicolumn{2}{|c|}{ Overweight (33.3\%) } & \multicolumn{2}{|c|}{ Obese $(13.4 \%)$} \\
\hline & Years & $95 \% \mathrm{Cl}$ & Years & $95 \% \mathrm{Cl}$ & Years & $95 \% \mathrm{Cl}$ & Years & $95 \% \mathrm{Cl}$ \\
\hline \multicolumn{9}{|l|}{ Overall } \\
\hline TLE & $15 \cdot 75$ & $15 \cdot 32,16 \cdot 18$ & $12 \cdot 37$ & $11.53,13.21$ & 15.94 & $15.45,16.43$ & 14.66 & $14 \cdot 13,15 \cdot 19$ \\
\hline HLE & $10 \cdot 63$ & $10 \cdot 36,10 \cdot 9$ & 8.17 & $7.35,8.99$ & 9.84 & $9.53,10.15$ & 8.08 & $7.69,8.47$ \\
\hline UHLE & $5 \cdot 12$ & $4.85,5.39$ & 4.20 & $3.53,4.87$ & $6 \cdot 11$ & $5.78,6.44$ & $6 \cdot 58$ & $6 \cdot 17,6.99$ \\
\hline \multicolumn{9}{|c|}{ Initial state } \\
\hline \multicolumn{9}{|c|}{ Good health } \\
\hline TLE & $15 \cdot 18$ & $14.96,15.40$ & $12 \cdot 23$ & $11.56,12.9$ & $15 \cdot 20$ & $14.93,15.47$ & $14 \cdot 31$ & $13.94,14.68$ \\
\hline HLE & $11 \cdot 26$ & $11.06,11.46$ & 9.18 & $8.49,9.87$ & $10 \cdot 57$ & $10 \cdot 33,10 \cdot 81$ & $9 \cdot 16$ & $8.83,9.49$ \\
\hline UHLE & 3.92 & $3.76,4.08$ & 3.05 & $2.62,3.48$ & 4.62 & $4 \cdot 40,4 \cdot 84$ & $5 \cdot 15$ & $4 \cdot 86,5 \cdot 44$ \\
\hline \multicolumn{9}{|c|}{ Poor health } \\
\hline TLE & 13.07 & $12 \cdot 82,13 \cdot 32$ & 9.24 & $8.48,10.00$ & 13.51 & $13 \cdot 20,13 \cdot 83$ & 12.63 & $12.20,13.06$ \\
\hline HLE & 5.93 & $5 \cdot 69,6 \cdot 17$ & 3.54 & $2 \cdot 87,4 \cdot 21$ & 5.54 & $5 \cdot 29,5.79$ & 4.63 & $4.30,4.96$ \\
\hline UHLE & $7 \cdot 15$ & $6.95,7.35$ & 5.70 & $5 \cdot 15,6 \cdot 25$ & 7.97 & $7.73,8.21$ & 8.00 & $7.67,8.33$ \\
\hline
\end{tabular}

Table 2. Risk ratios for the effect of BMI on transitioning between health states and death

(Risk ratios (RR) and $95 \%$ confidence intervals)

\begin{tabular}{|c|c|c|c|c|c|c|c|}
\hline & \multirow{2}{*}{$\frac{\text { Normal weight }(50.1 \%)}{\mathrm{RR}}$} & \multicolumn{2}{|c|}{ Underweight (3.2\%) } & \multicolumn{2}{|c|}{ Overweight $(33.3 \%)$} & \multicolumn{2}{|c|}{ Obese $(13.4 \%)$} \\
\hline & & $\mathrm{RR}$ & $95 \% \mathrm{Cl}$ & $\mathrm{RR}$ & $95 \% \mathrm{Cl}$ & $\mathrm{RR}$ & $95 \% \mathrm{Cl}$ \\
\hline Good health $\rightarrow$ poor health (incidence) & Ref. & 1.19 & $0.97,1.44$ & 1.15 & $1.08,1.23$ & 1.60 & $1.46,1.75$ \\
\hline Good health $\rightarrow$ death & Ref. & $1 \cdot 30$ & $0 \cdot 77,2 \cdot 20$ & $1 \cdot 11$ & $0.93,1.32$ & 0.98 & $0.72,1.34$ \\
\hline Poor health $\rightarrow$ good health (recovery) & Ref. & 0.82 & $0.63,1.06$ & 0.93 & $0.85,1.02$ & 0.93 & $0.83,1.04$ \\
\hline Poor health $\rightarrow$ death & Ref. & 1.90 & $1.58,2 \cdot 28$ & 0.84 & $0.76,0.91$ & 0.95 & $0.85,1.06$ \\
\hline
\end{tabular}

Ref., referent values.

The results of the secondary analysis were not statistically significant, and are included in the online Supplementary material. A total of 1486 women were identified as obese at survey 1 , and of these 229 decreased their BMI class at survey 2
(222 became overweight, seven became normal weight and no obese women became underweight). Overall, women whose BMI decreased tended to have slightly higher TLE and HLE. When stratified by initial health state, the biggest difference 
identified was for women who began the survey in poor health, with almost 1 year of additional HLE added for women who lost weight compared with women who did not. Although this result was not statistically significant because of the relatively small number of obese women who lost weight, the result is clinically significant.

\section{Discussion}

We found that, compared with normal-weight women, overweight women at the age of 75 years could expect to have almost one more unhealthy year (0.99; $95 \%$ CI $0.56,1.42$ years) and fewer healthy ones $(0.79 ; 95 \%$ CI $0.37,1.21$ years). However, TLE at the age of 75 years for overweight women was not significantly longer than that for normal-weight women. There was a $16 \%$ reduction in the risk of death for overweight women who started the study in poor health, but those who started the study in good health had no survival advantage. Indeed, these women were $15 \%$ more likely to transition to poor health. Therefore, although being overweight may be slightly protective against death, especially for older women in poor health, it puts all women at a greater risk of poor health in later life, increasing unhealthy years and reducing healthy ones.

Obese women not only had significantly more unhealthy years $(1.46 ; 95 \%$ CI $0.97,1.95$ years) and fewer healthy years (2.55; 95\% CI 2.07, 3.03 years) but also lived 1 year less (1.09; $95 \%$ CI $0.41,1.77$ years), although the increase in TLE appeared to be confined to those initially in good health.

It is clear from our results that there is generally no benefit from being obese, as there was no significant protective effect for death (Table 2), and obesity was associated with a $60 \%$ increased risk of poor health (compared with normal-weight women). These findings support the work by DeCaria et al. ${ }^{(35)}$, who hypothesised that as obesity is associated with several diseases that increase functional disability, the increasing prevalence of obesity will lead to more unhealthy years of life, and greater health-care costs.

It has been suggested that extra weight over a certain age is protective against mortality because of its protective effects against osteoporotic fractures and cognitive decline, and the fact that it may act as an energy reserve, which protects proteinenergy malnutrition ${ }^{(36)}$. In our study, this benefit was not observed to extend to women who were obese, perhaps because obesity may have an impact on a person's physical activity level, which is known to have a positive effect on mortality ${ }^{(37)}$. Overweight older people may still be able to move well and engage in physical activity, and therefore they are able to reap both the benefits of additional weight and physical activity. Thus, it may be that beyond a certain excess of weight the benefit may be negated by the concurrent reductions in physical activity. Obesity is also associated with high levels of metabolic dysfunction and chronic disease, which will also affect disability and self-rated health ${ }^{(38)}$

Finally, our results support the literature that being underweight is linked to poorer health outcomes. Underweight women had the shortest TLE and HLE at 75 years, and were almost twice as likely to die from a state of poor health as normal-weight women. It is possible that this relationship could reflect underlying pre-existing terminal diseases for this BMI group, although results from the sensitivity analysis showed no substantive differences. Interestingly, underweight women had the shortest UHLE; however, this is probably a reflection of their overall shorter TLE, rather than being an advantage per se. For example, underweight women may die faster following disease onset, and thus spend fewer years in an unhealthy state.

This study demonstrates the importance of entering old age in a state of good self-rated health. Regardless of BMI status, TLE and HLE at 75 years were greater for those whose initial health status was good. In addition, no BMI group showed a significant benefit in terms of recovery (transitioning from poor to good health), and no greater risk of death if the initial health status was good.

One limitation of this study is the lack of adjustment for possible confounding variables, such as disease or physical activity. However, as yet this software cannot adjust for confounding factors but rather calculates HLE within the separate strata, which can result in small subgroup sizes. Future work will also investigate the effect of physical activity on TLE and HLE, and the interaction between physical activity and BMI. Another limitation of the study is that BMI was calculated from self-reported height and weight, with potential for underestimation of weight and BMI category ${ }^{(39)}$.

The secondary analysis examined weight reduction in the obese group. Although these results were not statistically significant, the increase in HLE of 1 year for obese women who started the study in poor health and who lost weight is clinically significant. Our results suggest that the greatest benefits of weight loss in the obese elderly may be achieved for those who are in poor health, whereas only modest gains may be achieved for those who are in good health. However, one complication of looking at BMI change as opposed to baseline BMI is that it can be difficult to differentiate between intentional weight loss (e.g., for obesity-related disorders) and unintentional weight loss (as a result of ill health) ${ }^{(12)}$. Dahl et $a l^{(2)}$ found that, compared with older persons whose BMI remained stable, the mortality hazard was $65 \%$ higher for those whose BMI reduced over time, and $53 \%$ higher for those whose BMI increased over time. In addition to the small sample size, the inability to identify intentional weight loss may have contributed to the non-significant findings of the secondary analysis. Weight loss in older adults (aged 70-79 years) can be associated with the loss of lean muscle mass ${ }^{(40)}$. However, Felix \& West $^{(15)}$ state that those obese older adults who are likely to be prescribed weight loss are also more likely to have increased lean mass and bone density, making any potential loss in these areas less of a concern. Further, weight loss in obese older adults has also been associated with improved physical function ${ }^{(41,42)}$, reduced pain $^{(43)}$ and improved mental state and quality of life ${ }^{(44)}$. In addition, many of these results have been shown to be enhanced when combined with exercise ${ }^{(42-45)}$.

The relationship between weight and HLE has important implications for nutrition for older people. Concerns are for obesity, which has serious implications for the older person's health and quality of life, as well as increasing demands for health services, and for underweight which is very common in older age. Maintenance of lean body mass is important in both 
scenarios. Because of the possible complications of weight loss in obese older adults, it has been recommended that weight-loss intervention be individualised based on weight-loss history and medical conditions ${ }^{(13)}$. We support the concept of individualised assessment, and further suggest that the benefits of a weight-loss intervention may not outweigh the risks for those obese older adults in good health, but that it may be beneficial for obese older adults in poor health. Many older people have insufficient dietary intakes of protein and other nutrients to maintain muscle mass, particularly those with chronic conditions. It is important that obese older persons who are prescribed weight loss, as well as underweight older adults, are provided with sufficient dietary advice with the aim of maintaining lean muscle mass. Older people also have reduced levels of physical activity, which further contributes to loss of muscle mass and strength ${ }^{(46,47)}$. Under-nutrition is also determined by disease and psychosocial factors including living alone ${ }^{(48)}$.

The study used self-rated health, which has been found to be a significant predictor of survival in older Australians and other older populations, and may capture the complex and interacting nature of multiple diseases and varying disease severity among older persons, including frailty, and the subjective value the person places on their quality of life and well-being ${ }^{(49)}$.

\section{Conclusion}

This study is significant in determining the effect of BMI on HLE among old women. Although an overweight BMI was associated with slightly reduced mortality among those in poor health (compared with normal weight), among those in good health this excess weight increased the risk of poor health and resulted in fewer years spent healthy and more years unhealthy. Further, being obese had no beneficial effect on mortality, and it greatly increased the risk of poor health. However, weight loss in obese women may be beneficial in increasing HLE for those in poor health. Being underweight was associated with increased mortality. Finally, regardless of BMI, we show that it is important to enter old age in a state of good health. In a time when life expectancy continues to increase, it is important to discover whether these years are spent in good or poor health, as this has implications for both health-care utilisation and for the quality of life older persons can expect. It is therefore recommended that older women maintain a healthy weight, and that public health initiatives continue to promote health and prevent disease and disability in younger adults to ensure that as many as possible enter old age in good health.

\section{Acknowledgements}

The research on which this paper is based was conducted as part of the Australian Longitudinal Study on Women's Health, the University of Newcastle and the University of Queensland. The authors would like to thank Nicolas Brouard, senior researcher at the Mortality, Health and Epidemiology Research Unit, Institut National d'Etudes Démographiques, for his technical advice on the software IMaCh.
The authors are grateful to the Australian Government Department of Health for funding and to the women who provided the survey data. This research was supported by infrastructure and staff of the Research Centre for Generational Health and Ageing, who are members of the Hunter Medical Research Institute. The authors acknowledge the assistance of the Data Linkage Unit at the Australian Institute of Health and Welfare (AIHW) for undertaking the data linkage to the NDI.

L. L. contributed to drafting all sections of the paper, prepped the paper for submission, performed data preparation and analysis and contributed to the literature review, interpretation of results and discussion; J. E. B. contributed to editing and critique of the entire paper, provided substantive expertise and contributed to the literature review, interpretation of results and discussion; C. J. contributed to editing and critique of the entire paper, provided substantive expertise, provided statistical expertise and contributed to the literature review, interpretation of results and discussion.

The authors declare that there are no conflicts of interest.

\section{Supplementary material}

For supplementary material/s referred to in this article, please visit http://dx.doi.org/10.1017/S0007114516002403

\section{References}

1. World Health Organization (2000) Obesity - Preventing and Managing the Global Epidemic. Geneva: WHO.

2. Dahl AK, Fauth EB, Ernsth-Bravell M, et al. (2013) Body mass index, change in body mass index, and survival in old and very old persons. J Am Geriatr Soc 61, 512-518.

3. Diehr PH, O'Meara ES, Fitzpatrick A, et al. (2008) Weight, mortality, years of healthy life, and active life expectancy in older adults. J Am Geriatr Soc 56, 76-83.

4. Singh PN, Haddad E, Tonstad S, et al. (2011) Does excess body fat maintained after the seventh decade decrease life expectancy? J Am Geriatr Soc 59, 1003-1011.

5. Nagai M, Kuriyama S, Kakizaki M, et al. (2012) Impact of obesity, overweight and underweight on life expectancy and lifetime medical expenditures: the Ohsaki Cohort Study. BMJ Open 2, e000940.

6. Winter JE, MacInnis RJ, Wattanapenpaiboon N, et al. (2014) BMI and all-cause mortality in older adults: a meta-analysis. Am J Clin Nutr 99, 875-890.

7. Flegal KM, Kit BK, Orpana H, et al. (2013) Association of allcause mortality with overweight and obesity using standard body mass index categories. JAMA 309, 71-82.

8. Stessman J, Jacobs JM, Ein-Mor E, et al. (2009) Normal body mass index rather than obesity predicts greater mortality in elderly people: the Jerusalem Longitudinal Study. J Am Geriatr Soc 57, 2232-2238.

9. Flicker L, McCaul KA, Hankey GJ, et al. (2010) Body mass index and survival in men and women aged 70 to 75 . J Am Geriatr Soc 58, 234-241.

10. Prospective Studies Collaboration, Whitlock G, Lewington S, et al. (2009) Body-mass index and cause-specific mortality in 900000 adults: collaborative analyses of 57 prospective studies. Lancet 373, 1083-1096.

11. Roberson LL, Aneni EC, Maziak W, et al. (2014) Beyond BMI: the 'metabolically healthy obese' phenotype and its association with clinical/subclinical cardiovascular disease and 
all-cause mortality - a systematic review. BMC Public Health 14, 14 .

12. Elia M (2001) Obesity in the elderly. Obes Res 9, Suppl. 4, 244S-248S.

13. Bales CW \& Buhr G (2008) Is obesity bad for older persons? A systematic review of the pros and cons of weight reduction in later life. J Am Med Dir Assoc 9, 302-312.

14. Han TS, Tajar A \& Lean MEJ (2011) Obesity and weight management in the elderly. Br Med Bull 97, 169-196.

15. Felix HC \& West DS (2013) Effectiveness of weight loss interventions for obese older adults. Am J Health Promot 27, 191-199.

16. Collerton J, Barrass K, Bond J, et al. (2007) The Newcastle 85+ study: biological, clinical and psychosocial factors associated with healthy ageing: study protocol. BMC Geriatr 7, 14 .

17. Robine JM \& Jagger C (2005) The relationship between increasing life expectancy and healthy life expectancy. Ageing Horiz 3, 14-21.

18. Christensen K, Doblhammer G, Rau R, et al. (2009) Ageing populations: the challenges ahead. Lancet 374, 1196-1208.

19. Robine JM, Saito Y \& Jagger C (2009) The relationship between longevity and healthy life expectancy. Qual Ageing 10, 5-14.

20. Jagger C, Gillies C, Moscone F, et al. (2008) Inequalities in healthy life years in the 25 countries of the European Union in 2005: a cross-national meta-regression analysis. Lancet $\mathbf{3 7 2}$, 2124-2131.

21. Jagger C, Matthews R, Matthews FE, et al. (2007) The burden of diseases on disability-free life expectancy in later life. I Gerontol A Biol Sci Med Sci 62A, 408-414.

22. Crimmins EM, Hayward MD, Hagedorn A, et al. (2009) Change in disability-free life expectancy for Americans 70 years old and older. Demography 46, 627-646.

23. Steensma C, Loukine L, Orpana H, et al. (2013) Comparing life expectancy and health-adjusted life expectancy by body mass index category in adult Canadians: a descriptive study. Popul Health Metr 11, 21.

24. Reynolds SL, Saito Y \& Crimmins EM (2005) The impact of obesity on active life expectancy in older American men and women. Gerontologist 45, 438-444.

25. Byles JE, Dobson AJ, Pachana NA, et al. (2010) Major report E. Women, health and ageing: findings from the Australian Longitudinal Study on Women's Health. Women's Health Australia. http://www.alswh.org.au/publications-and-reports/ major-reports (accessed June 2016).

26. Brown W, Bryson L, Byles JE, et al. (1998) Women's Health Australia: recruitment for a national longitudinal cohort study. Women Health 28, 23-40.

27. Lee C, Dobson AJ, Brown WJ, et al. (2005) Cohort profile: the Australian Longitudinal Study on Women's Health. Int J Epidemiol 34, 987-991.

28. Dobson A, Hockey R, Brown WJ, et al. (2015) Cohort profile update: Australian Longitudinal Study on Women's Health. Int J Epidemiol 44, 1547, 1547a-1547f.

29. World Health Organization (2015) Obesity and overweight. Fact sheet 311. http://www.who.int/mediacentre/factsheets/ fs311/en/ (accessed June 2016).

30. Powers J, Ball J, Adamson L, et al. (2000) Effectiveness of the National Death Index for establishing the vital status of older women in the Australian Longitudinal Study on Women's Health. Aust N Z J Public Health 24, 526-528.
31. Lièvre A, Brouard N \& Heathcote C. (2003) The estimation of health expectancies from cross-longitudinal surveys. Math Popul Stud 10, 211-248.

32. Willekens K \& Putter H (2014) Software for multistate analysis. Demogr Res 31, 381-420.

33. Jackson CH (2011) Multi-state models for panel data: the msm package for R. J Stat Softw 38, 1-28.

34. Jones M, Hockey R, Mishra GD, et al. (2014) Visualising and modelling changes in categorical variables in longitudinal studies. BMC Med Res Methodol 14, 1-8.

35. DeCaria JE, Sharp C \& Petrella RJ (2012) Scoping review report: obesity in older adults. Int $J$ Obes (Lond) $\mathbf{3 6}$, $1141-1150$

36. Oreopoulos A, Kalantar-Zadeh K, Shrma AM, et al. (2009) The obesity paradox in the elderly: potential mechanisms and clinical implications. Clin Geriatr Med 25, 643-659.

37. Gregg EW, Cauley JA, Stone KL, et al. (2003) Relationship of changes in physical activity and mortality among older women. J Am Med Assoc 289, 2379-2386.

38. Burton B, Foster W, Hirsch J, et al. (1985) Health implications of obesity: an NIH Consensus Development Conference. Int J Obes $9,155-170$

39. Stommel M \& Schoenbprn C (2009) Accuracy and usefulness of BMI measures based on self-reported weight and height: findings from the NHANES and NHIS 2001-2006. BMC Public Health 9, 421

40. Newman AB, Lee JS, Visser M, et al. (2005) Weight change and the conservation of lean mass in old age: the Health, Aging and Body Composition Study. Am J Clin Nutr 82, 872-878.

41. Sartorio A, Lafortuna CL, Agosti F, et al. (2004) Elderly obese women display the greatest improvement in stair climbing performance after a 3-week body mass reduction program. Int J Obes Relat Metab Disord 28, 1097-1104.

42. Villareal DT, Banks M, Sinacore DR, et al. (2006) Effect of weight loss and exercise on frailty in obese older adults. Arch Intern Med 166, 860-866.

43. Messier SP, Loeser RF, Miller GD, et al. (2004) Exercise and dietary weight loss in overweight and obese older adults with knee osteoarthritis. Arthritis Rheum 50, 1501-1510.

44. Napoli N, Shah K, Waters DL, et al. (2014) Effect of weight loss, exercise, or both on cognition and quality of life in obese older adults. Am J Clin Nutr 100, 189-198.

45. Bouchonville M, Armamento-Villareal R, Shah K, et al. (2014) Weight loss, exercise, or both and cardiometabolic risk factors in obese older adults: results of a randomized controlled trial. Int J Obes 38, 423-431.

46. Mithal A, Bonjour JP, Boonen S, et al. (2013) Impact of nutrition on muscle mass, strength, and performance in older adults. Osteoporos Int 24, 1555-1566.

47. Morris MS \& Jacques PF (2013) Total protein, animal protein and physical activity in relation to muscle mass in middle-aged and older Americans. Br J Nutr 109, 1294-1303.

48. Schilp J, Wijnhoven HAH, Deeg DJH, et al. (2011) Early determinants for the development of undernutrition in an older general population: Longitudinal Aging Study Amsterdam. Br J Nutr 106, 708-717.

49. McCallum J, Shadbolt B \& Wang D (1994) Self-rated health and survival: a 7-year follow-up study of Australian elderly. Am J Public Health 84, 1100-1105. 